\section{TRANS-3-(METHYLTHIO)- ACRYLIG ACID, A NEW METABOLIC PRODUCT FROM STREPTOMYCES LINCOLNENSIS}

\section{J. Visser and H. F. Meyer}

The Upjohn Company, Kalamazoo, Michigan, U.S.A.

(Received for publication August 23, 1969)

During development of the lincomycin* fermentation, we studied the effect of the addition of $d l$ - and $l$-methionine to the culture medium. The microorganism used for these experiments was a mutant of Streptomyces lincolnensis var. lincolnensis $\mathrm{sp}$. n. ${ }^{1)}$ The broth obtained in this way showed a significantly higher potency with a chemical assay method ${ }^{2)}$ than with the microbiological assay (Sarcina lutea) $)^{3)}$, as shown in Fig. 1. The chemical assay is based on a colorimetric determination of methyl mercaptan generated from lincomycin on acid hydrolysis ${ }^{4}$.

The gas mixture obtained by hydrolysis of filtered broth was analyzed by vapor phase chromatography ${ }^{5}$. Methyl mercaptan was the only sulfur-containing compound present. Methionine itself does not form methyl mercaptan under the experimental conditions used. Therefore, methyl mercaptan originated not only from the linco-

Fig. 1. Comparison of colorimetric and microbiological assays of lincomycin with and without added $d l$-menthionine (2 g/liter).

Assays expressed as lincomycin base (mol. weight 406) and as methylthioacrylic acid (mol. weight 118).

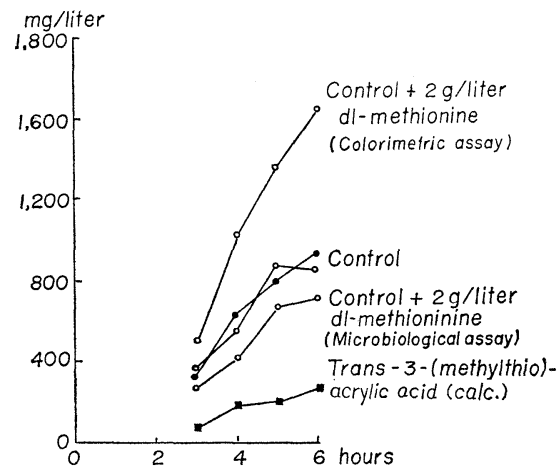

mycin, but also from at least one other compound with a lower (or no) bioactivity.

This material was isolated in the following manner: First, the filtered broth was extracted with $n$-butanol at $\mathrm{pH} 2.5$. Then the chemically active compound was reextracted into water at $\mathrm{pH} 10$. Traces of lincomycin were removed by extraction with methylene chloride. The remaining aqueous solution was adjusted to $\mathrm{pH} 7$ and evaporated to $1 / 10$ of its volume. Addition of ten volumes of acetone precipitated impurities as an oil, which was discarded. The remaining solution on concentration gave crude crystals which were recrystallized successively from acetone/water, ethanol/water, and ethanol.

The purified compound melted at $140^{\circ} \mathrm{C}$ and was identified by IR, NMR, mass spectrum, and elemental analysis as trans3-(methylthio)-acrylic acid. Analysis calculated for $\mathrm{C}_{4} \mathrm{H}_{6} \mathrm{O}_{2} \mathrm{~S}$ (in percent): C 40.66, $\mathrm{H} 5.12$, O 27.08, S 27.14; found: C 40.84, H 5.09, O 26.90, S 25.28. Molecular weight: 118 (mass spectrum). Chemical shifts and their assignment in $\delta$ (rel. TMS) of an NMR spectrum at $60 \mathrm{mc}$ in $\mathrm{d}_{6}$ acetone: $\mathrm{CH}_{3}$ 2.40, $\mathrm{COOH} 9.58$, olefinic $\mathrm{H} 5.82$ and 7.80 , doublets with $\mathrm{J}=15 \mathrm{cps}$ (indicates transconfiguration).

Fig. 1 shows the calculated course of synthesis of this compound during the fermentation.

\section{Acknowledgement}

We thank Dr. H. K. JAHNKE and co-workers for assays and vapor phase chromatograms, Dr. G. SLOMP and co-workers for spectral data.

\section{References}

1) Mason, D.J.; A. Dietz \& C. Deboer: Lincomycin, a new antibiotic. I. Discovery and biological properties. Antimicr. Agents \& Chemoth.-1962 : 554 569, 1963

2) Рволсотт, G. C.: Automated assay for the antibiotic lincomycin. J. Pharmaceut. Sci. $55: 423 \sim 425,1966$

3) Hanka, L. J.; D. J. Mason, M. R. Burch \& R. W. Treick: Lincomycin, a new antibiotic. Microbiological assay. Antimicr. Agents \& Chemoth.-1962:565 569, 1963

4) Hoeksema, H.; B. Bannister, R. D. BirkenMeYer, F. Kagan, B. J. Magerlein, F. A. McKellar, W. Schroeder, G. Slomp \& R. R. Herr: Chemical studies on lincomycin. I. The structure of lincomycin. J. Am. Chem. Soc. $86: 4223 \sim 4224,1964$

5) Farrugia, V. J. \& C. L. Jarreau: Separation of isopropyl alcohol from aliphatic sulfides and thiols by gas chromatography. Anal. Chem. $34: 271 \sim 273,1962$ 